\title{
Diabetes Mellitus e Transtorno Depressivo: a bidirecionalidade entre duas doenças crônicas
}

\author{
Diabetes Mellitus and Depressive Disorder: the bidirectionality between two chronic \\ diseases
}

\section{Diabetes Mellitus y Trastorno Depresivo: la bidireccionalidad entre dos enfermedades crónicas}

Renata de Souza Jardim ${ }^{1 *}$, Barbara Mafalda Robbiati ${ }^{2}$, Brenda Akemi Gaspi Okamoto ${ }^{3}$, Edivaldo Pereira de Campos Junior ${ }^{4}$, Fernanda Luz Neves da Rocha ${ }^{2}$, Juliana Guiotti ${ }^{5}$, Nathalia Di Mase Salgado ${ }^{6}$, Lorenzo Guerrero ${ }^{7}$, Mônica Isaura Corrêa².

\section{RESUMO}

Objetivo: Discutir a associação entre a prevalência de depressão em pacientes diabéticos, avaliando aspectos epidemiológicos, psicossociais, fisiopatológicos e o correto manejo clínico. Revisão bibliográfica: Observou-se uma ligação bidirecional entre diabetes e depressão, sendo esta quase duas vezes mais prevalente em pacientes com diabetes tipo 2. Constatações corroboradas pela principal hipótese fisiopatológica encontrada, que une os dois distúrbios, na qual o aumento sustentado de catecolaminas na doença depressiva levaria ao aumento da glicemia, através da diminuição da síntese de insulina ou aumento da resistência periférica a ação da insulina, além dos efeitos neuroquímicos do diabetes sobre os sistemas centrais serotoninérgicos, noradrenérgicos e dopaminérgicos, diminuindo a função destas aminas. Percebese ainda a relevância psicossocial dos distúrbios presentes de forma concomitante, associados a uma baixa qualidade de vida, adesão reduzida às prescrições e autogestão, e maior impacto e prevalência de complicações diabéticas, ocasionando um aumento no uso e, consequentemente, nos custos de saúde. Considerações finais: Apesar da clara correlação encontrada entre tais patologias não existem grandes artigos com grupo controle e estudos randomizados que confirmem tal associação.

Palavras-chave: Diabetes mellitus, Transtorno depressivo, Doença crônica.

\begin{abstract}
Objective: Discuss the association between the prevalence of depression in diabetic patients, evaluating epidemiological, psychosocial, pathophysiological aspects and the correct clinical management. Bibliographic review: There was a bidirectional link between diabetes and depression, which is almost twice as prevalent in patients with type 2 diabetes. Findings corroborated by the main pathophysiological hypothesis found, which joins the two disorders, in which the sustained increase in catecholamines in depressive illness would lead to an increase in blood glucose, through a decrease in insulin synthesis or an increase in peripheral resistance to the action of insulin, in addition to the neurochemical effects of diabetes on the central serotonergic, noradrenergic and dopaminergic systems, decreasing the function of these amines. The psychosocial relevance of the disorders present in a concomitant manner, associated with a low quality of life, reduced adherence to prescriptions and self-management, and a greater impact and prevalence of diabetic
\end{abstract}

${ }^{1}$ Centro Universitário Católico Salesiano Auxilium (UniSALESIANO), Araçatuba - SP.

*E-mail: renatasj2013@gmail.com

2 Centro Universitário de Caratinga (UNEC), Caratinga - MG.

${ }^{3}$ Faculdade de Medicina de Olinda (FMO), Olinda - PE.

${ }^{4}$ Universidad de Buenos Aires (UBA), Buenos Aires - AR.

${ }^{5}$ Fundação Educacional do Município de Assis (FEMA), Assis - SP.

${ }^{6}$ Centro Universitário das Américas (FAM), São Paulo - SP.

${ }^{7}$ Centro Universitário Redentor (UNIREDENTOR), Itaperuna - RJ.

SUBMETIDO EM: 4/2021

ACEITO EM: 5/2021

PUBLICADO EM: 6/2021 
complications is also perceived, causing an increase in the use and, consequently, in the costs of health. Final considerations: Despite the clear correlation found between such pathologies, there are no large articles with a control group and randomized studies that confirm this association.

Key words: Diabetes mellitus, Depressive disorder, Chronic disease.

\section{RESUMEN}

Objetivo: Discutir la asociación entre la prevalencia de depresión en pacientes diabéticos, evaluando aspectos epidemiológicos, psicosociales, fisiopatológicos y el correcto manejo clínico. Revisión bibliográfica: Hubo un vínculo bidireccional entre diabetes y depresión, que es casi el doble de prevalente en pacientes con diabetes tipo 2. Hallazgos corroborados por la principal hipótesis fisiopatológica encontrada, que une los dos trastornos, en los que el aumento sostenido de catecolaminas en la enfermedad depresiva conduciría a un aumento de la glucosa en sangre, por una disminución de la síntesis de insulina o un aumento de la resistencia periférica a la acción de insulina, además de los efectos neuroquímicos de la diabetes sobre los sistemas central serotoninérgico, noradrenérgico y dopaminérgico, disminuyendo la función de estas aminas. También se percibe la relevancia psicosocial de los trastornos presentes de manera concomitante, asociados a una baja calidad de vida, menor adherencia a la prescripción y autocuidado, y un mayor impacto y prevalencia de las complicaciones diabéticas, provocando un incremento en el uso y, en consecuencia, en los costos de salud. Consideraciones finales: Apesar de la clara correlación encontrada entre este tipo de patologías, no existen grandes artículos con grupo control y estudios aleatorizados que confirmen esta asociación.

Palabras clave: Diabetes mellitus, Trastorno depresivo, Enfermedad crónica.

\section{INTRODUÇÃO}

O Diabetes Mellitus (DM) faz parte de um distúrbio metabólico caracterizado por hiperglicemia crônica, decorrente de uma secreção insuficiente de insulina pelas células beta pancreáticas, por uma resposta do tecido diminuída à insulina ou ambos combinados. Tem uma prevalência de 285 milhões de pessoas $(6,6 \%$ da população mundial) sendo afetadas (FIORE V, et al., 2014). Já no Brasil, afeta aproximadamente $7,6 \%$ da população brasileira entre 30 e 69 anos (MOREIRA R, et al., 2003). Além disso, o DM está associado a complicações microvasculares como retinopatia diabética, nefropatia diabética e neuropatia diabética, e também a complicações macrovasculares como doenças cardiovasculares. Evidências epidemiológicas atuais sugerem a associação do DM com a depressão, um transtorno psíquico caracterizado por alteração patológica do humor, com consequente comprometimento das atividades sociais (FIORE V, et al., 2014).

A depressão é altamente prevalente, estima-se que acometa $3 \%$ a $5 \%$ da população geral. Já em populações clínicas, a incidência é ainda maior, pois a depressão é encontrada em $5 \%$ a $10 \%$ dos pacientes ambulatoriais e $9 \%$ a $16 \%$ de internados. Entretanto, somente $35 \%$ dos doentes são diagnosticados e tratados adequadamente (TENG CT, et al., 2005). Estima-se que 5-12\% dos homens e 10-15\% das mulheres irão vivenciar um transtorno depressivo maior durante a vida, com uma síndrome clínica evidente em 3\% e 5-9\%, respectivamente (FIORE V, et al., 2014).

De acordo com o Diagnostic and Statistical Manual of Mental Disorders 5 (DSM-IV), os critérios para o diagnóstico de transtorno depressivo maior (TDM) consistem na presença quase todos os dias e por 2 semanas, de, pelo menos, cinco dos seguintes sintomas: humor deprimido, interesse ou prazer nitidamente reduzido em todas ou quase todas as atividades, perda ou ganho significativo de peso ou apetite diminuído ou aumentado, insônia ou hipersonia, agitação ou retardo psicomotor, fadiga ou perda de energia, sentimentos de inutilidade ou culpa, diminuição da capacidade de concentração ou de tomada de decisões, pensamentos recorrentes de morte ou suicídio. Para diagnosticar a depressão primária, as condições depressivas devidas a outras doenças, a certos medicamentos ou luto, devem ser descartadas (MUSSELMAN D, et al., 2003).

Ao longo dos anos, vários estudos analisaram a relação do DM com sintomas depressivos, de forma que foi possível estabelecer a bidirecionalidade entre essas duas doenças crônicas. Em média, 20 a 30\% dos 
pacientes diabéticos também sofrem de transtornos depressivos, dos quais $10 \%$ são do tipo TDM (FIORE V, et al., 2014). Anderson RJ, et al. (2001), concluíram através de uma meta-análise, que o DM praticamente duplica o risco de depressão. Um em cada três pacientes diabéticos tem depressão e mulheres diabéticas apresentam maior risco de desenvolverem esta patologia (MOREIRA R, et al., 2003). Além disso, é comum a recorrência de depressão em pacientes diabéticos após a remissão em até $80 \%$ dos pacientes tratados em um período de cinco anos, e alguns autores consideram que a depressão está muito relacionada com o número e com a gravidade das complicações diabéticas (FIORE V, et al., 2014).

A associação entre depressão e diabetes é muito frequente, deteriorando tanto o quadro psíquico, quanto a doença de base. Em termos fisiopatológicos, a depressão é responsável por modificações hormonais e neuroquímicas que levam a efeitos hiperglicemiantes e podem resultar em alterações no metabolismo glicêmico. Por outro lado, o DM apresenta ação neuroquímica sobre sistemas serotoninérgicos, noradrenérgicos e dopaminérgicos, resultando em diminuição da função monoaminérgica (MOREIRA R, et al., 2003).

Vários fatores estão associados às taxas aumentadas de depressão em pacientes diabéticos, entre eles, o estilo de vida restrito, mudanças de hábitos alimentares, controle diário da glicemia e maior risco de desenvolver complicações (MOREIRA R, et al., 2003). Em contrapartida, pessoas com depressão também são mais propensas a desenvolver DM, principalmente por diminuição do autocuidado, comprometendo o controle glicêmico (FIORE V, et al., 2014).

Nesse sentido, o objetivo desta revisão bibliográfica foi discutir a associação entre a prevalência de depressão em pacientes diabéticos, avaliando aspectos epidemiológicos, psicossociais, fisiopatológicos e o correto manejo clínico.

\section{REVISÃO BIBLIOGRÁFICA}

\section{Epidemiologia e prevalência na sociedade}

Desde meados da década de 1980, vários estudos longitudinais e transversais determinaram a associação entre diabetes e TDM, sendo este último uma modificação patológica do humor. É necessário para diagnóstico de TDM, que dentre os sintomas apresentados pelo paciente, estejam pelo menos o humor deprimido ou a perda de interesse ou prazer. As evidências epidemiológicas atuais sugerem que as pessoas com diabetes têm um risco maior de sofrerem de uma condição depressiva durante a vida (FIORE V, et al., 2014).

A taxa de depressão é significativamente mais alta em pacientes diabéticos e consideravelmente maior em idosos. De $20 \%$ a $30 \%$ dos pacientes com diabetes (tanto do tipo 1 , quanto do tipo 2), sofrem de transtornos depressivos clinicamente relevantes. No entanto, cerca de dois terços dos pacientes não são diagnosticados corretamente e, consequentemente, tratados para sua síndrome depressiva, possivelmente devido à menor atenção dos médicos ao estado psicológico (FIORE V, et al., 2014).

A depressão em pacientes diabéticos é uma condição crônica e grave, que tende à recorrência após a remissão em até $80 \%$ dos pacientes tratados em um período de cinco anos. Alguns autores consideram a depressão como altamente preditiva do número e da gravidade das complicações diabéticas (FIORE V, et al., 2014).

De fato, de acordo com múltiplos estudos transversais de Lustman $\mathrm{P}$, et al. (2000), nota-se que os pacientes com diabetes e depressão comórbida, apresentam controle glicêmico pobre e maior prevalência de complicações múltiplas do diabetes como retinopatia, nefropatia, neuropatia, disfunção sexual e complicações macrovasculares, sendo a retinopatia e a nefropatia, as principais complicações relacionadas com os sintomas depressivos, possivelmente devido ao grande comprometimento funcional que ambas acarretam (ROY A e ROY M, 2001). Além disso, Gary TL, et al. (2000), mostraram que pacientes diabéticos com sintomas depressivos mais graves apresentaram níveis mais elevados de pressão arterial diastólica, colesterol total, Low Density Lipoproteins (LDL)-colesterol e triglicérides, além de valores mais baixos de High Density Lipoproteins (HDL)-colesterol. 
De acordo com Moreira RO, et al. (2015), os pacientes diabéticos com maior risco de depressão têm menos acesso à educação, são solteiros ou têm pouco suporte social e experiência crônica a estressores ou eventos negativos da vida. Segundo ele, a educação, o impacto funcional do diabetes e o estresse financeiro podem contribuir mais para a depressão e ansiedade em pacientes com diabetes no início da doença. Também existem diferenças de gênero: mulheres com diabetes são duas vezes mais propensas a sofrer psicopatia em relação aos homens.

A presença de depressão maior em pacientes diabéticos resulta em amplificação de sintomas, ou seja, mesmo quando controlada a gravidade do DM, os pacientes diabéticos com depressão comórbida apresentam mais sintomas associados ao diabetes do que seus colegas não deprimidos (CIECHANOWSKI $P$, et al., 2002).

É importante salientar, que de acordo com estudos de Ali S, et al. (2006), a associação de diabetes e depressão inclui um controle piorado da glicemia, uma falta de adesão ao tratamento, um controle metabólico deficiente e aumento do risco de complicações vasculares. Além disso, essa condição tem sido associada ao aumento da incapacidade e diminuição da qualidade de vida, elevação dos custos de saúde e até aumento do risco de mortalidade, representando um grande desafio para a saúde pública.

\section{Fisiopatologia da relação entre diabetes mellitus e depressão}

A fisiopatologia da relação entre diabetes tipo 2 e depressão, ainda não está totalmente clara. Contudo, há hipóteses em estudos que podem justificar esta associação. Uma das hipóteses mais encontrada na literatura é um aumento sustentado da concentração de catecolaminas na doença depressiva, o que leva ao aumento da glicemia através da diminuição da síntese de insulina ou aumento de resistência periférica à ação da insulina (MOREIRA R, et al., 2003). Por outro lado, o diabetes apresenta efeitos neuroquímicos sobre os sistemas centrais serotoninérgicos, noradrenérgicos e dopaminérgicos, que diminuem a função destas aminas (LEEDOM L, et al., 1991).

Outra justificativa que sustenta a relação entre DM e depressão, é que a hiperatividade do eixo hipotálamopituitária-adrenal e a ativação simpatomedular induzida por estresse mental em muitos pacientes com depressão maior, pode contribuir para a diminuição do transporte de glicose e da resistência à insulina. Isto acontece, pois a depressão faz com que os pacientes se tornem inativos, o que, combinado com níveis elevados de cortisol, causa adiposidade, obesidade e resistência à insulina. O tecido adiposo e os vasos danificados liberam citocinas pró-inflamatórias que induzem os sintomas depressivos da síndrome do "comportamento doentio", na qual prejudica a motivação do paciente para dar início a comportamentos saudáveis de autocuidado (RUSTAD JK, et al., 2011).

Pensava-se que o cérebro era insensível à insulina, porém, recentes descobertas apontam que a insulina e sua sinalização estão associadas à neuromodulação, neuroproteção e neurotransmissão de vários neurotransmissores importantes. A resistência do cérebro à insulina, que pode ser secundária à resistência periférica à insulina, tem sido associada a sintomas depressivos por meio de mudanças nas funções neuronais, tais como: déficit metabólico e energético, prejuízo na função do transportador de glicose-4, estresse oxidativo e do retículo endoplasmático, disfunção mitocondrial, acúmulo de produtos finais de glicação avançada (AGEs), espécies reativas de oxigênio (ROS) e espécies reativas de nitrogênio (RNS) com aumento na produção da neuroinflamação e ativação da cascata pró-apoptótica (MUKHERJEE $\mathrm{N}$ e CHATURVEDI SK, 2019).

Além disso, é importante salientar que o TDM e o diabetes tipo 2 compartilham anormalidades sobrepostas nas redes metabólicas definidas como sinalização de glicocorticóides, estresse oxidativo, biossíntese de energia, glicose-homeostase da insulina e processos inflamatórios (RUSTAD JK, et al., 2011).

\section{Associação entre DM e depressão}

A associação entre depressão e doenças clínicas é muito frequente, levando ao agravamento tanto do quadro psiquiátrico como da doença clínica, além de maior morbidade e mortalidade. Muitas vezes, a depressão é subdiagnosticada e subtratada, basicamente pela presença de sintomas depressivos, que podem ocorrer também em doenças crônicas (TENG CT, et al., 2005). 
Contudo, a prevalência da depressão é quase duas vezes maior em pacientes com diabetes tipo 2, e a associação entre diabetes e depressão simboliza um grande problema em consequência dos altos custos diretos e indiretos de saúde, e ao grande impacto na mortalidade e no risco cardiovascular. A associação entre DM e depressão é definida por uma baixa qualidade de vida, adesão reduzida às prescrições e autogestão, e maior impacto e prevalência de complicações diabéticas, ocasionando um aumento no uso e, consequentemente, nos custos com saúde. Além disso, a depressão tem um impacto negativo na atividade física, principalmente nos idosos, está associada a níveis estáveis de HbA1c (Hemoglobina Glicada) e intervém de maneira negativa no controle glicêmico por intermédio da interferência com a atividade do sistema nervoso central (FIORE V, et al., 2015).

Pacientes diabéticos com depressão apresentam um risco maior de obesidade, menor nível socioeconômico e educacional, como também um pior suporte social, sendo mais vulneráveis a estresses financeiros e psicossociais. Além do mais, pacientes com diabetes e depressão possuem um pior controle glicêmico e maior prevalência de várias complicações do diabetes, tais como: retinopatia, nefropatia, neuropatia, disfunção sexual e complicações macrovasculares (TENG CT, et al., 2005).

A ligação bidirecional entre DM e depressão pode ser explicada através do mecanismo compartilhado de patogênese de ambos os transtornos. Desregulação do eixo Hipotálamo-Pituitária-Adrenal (HPA), inflamação, leptina e fatores genéticos compartilhados, como os genes MC4R, NR3C1 e NR3C2, estão ligados para causar ambos os distúrbios. Outros fatores envolvidos na patogênese tanto do diabetes quanto da depressão, são a sinalização do cálcio e suas desregulações, proporcionando uma "ligação bidirecional" para estas doenças (MUKHERJEE N e CHATURVEDI SK, 2019).

À vista disso, identificar e tratar os transtornos depressivos é de suma importância em pacientes com diabetes, viabilizando um melhor manejo do DM, maior aceitação às prescrições e comportamentos sugeridos e uma melhora da qualidade de vida e do humor (FIORE V, et al., 2015).

\section{Tratamento}

As medicações antidepressivas são atualmente as mais prescritas ambulatorialmente, mas não existem evidências que confirmem o efeito desses medicamentos e o risco de desenvolver DM (FIORE V, et al., 2014).

De acordo com Nouwen A, et al. (2019), vários estudos longitudinais evidenciaram que a depressão no diabetes pode gerar autocuidado reduzido, declínio cognitivo aumentado e demência. Pacientes com depressão apresentam um humor alterado, anedonia, distúrbios do apetite e do sono, levando à diminuição da adesão à medicação por não conseguir seguir as prescrições, o que leva à piora dos sintomas e sentimento de frustração por não atingir o nível ideal do controle glicêmico.

Min YU, et al. (2014), relatam que na depressão maior ocorre uma alteração do eixo hipotálamo-hipófise adrenal devido ao aumento dos níveis do hormônio liberador de corticotrópico, gerando elevação do hormônio corticosteróide plasmático e como consequência, aumento da circunferência abdominal, elevação da concentração de ácidos graxos livres e aumento da frequência cardíaca, débito cardíaco e secreção de renina, juntamente com excesso de cortisol e androgênio, o que, por sua vez, pode levar ao DM.

\section{Psicofármacos e suas ações no diabetes e na depressão}

O uso dos tricíclicos foram associados com o aumento do apetite, aumento do peso e hiperglicemia, entretanto, a nortriptilina gerou diminuição dos valores da $\mathrm{HbA} 1 \mathrm{c}$ em pacientes diabéticos, no qual sugeriu que o tratamento dos sintomas depressivos pode levar à uma melhora indireta do controle metabólico (LUSTMAN PJ, et al., 1998).

Estudos com os inibidores seletivos de recaptação da serotonina (ISRS) mostraram efeitos positivos nos parâmetros do diabetes em pacientes com DM2. Entre os ISRS, a fluoxetina foi associada com melhoras dos níveis de $\mathrm{HbA1c}$, reduzindo a necessidade de insulina, contribuindo no emagrecimento e melhora na sensibilidade à insulina. Com a Sertralina, evidenciaram um progresso significativo dos sintomas depressivos e uma maior adesão às mudanças de estilo de vida (MEV) como exercício físico e dieta adequada, mas observou-se alterações discretas nos níveis de HbA1c. O Escitalopram apenas demonstrou uma melhora no 
controle glicêmico. Na classe dos inibidores da monoaminoxidase, foram verificados episódios de hipoglicemia e ganho de peso, sendo seu uso contraindicado para diabéticos (MAHEUX P, et al., 1997).

\section{Abordagens psicoterápicas no DM e depressão}

Segundo Fiore V, et al. (2014), a Terapia Cognitivo-Comportamental (TCC) proposta no tratamento de transtornos depressivos em pacientes diabéticos, juntamente com terapias medicamentosas, gerou uma melhora dos sintomas depressivos e do controle glicêmico. Contudo, Moreira RO, et al (2003), observaram que a TCC, a longo prazo, mesmo levando à melhora significativa no controle metabólico do paciente diabético, propicia dois fatores que podem levar a uma resposta insatisfatória ao tratamento: presença de complicações do DM e a má aderência à monitorização domiciliar da glicemia capilar. Os pacientes que apresentaram boa resposta à TCC não realizam uma monitorização domiciliar adequada do DM, tornando apenas o tratamento da depressão prioritário, reduzindo os cuidados com o diabetes (LUSTMAN PJ, et al.,1998).

\section{CONSIDERAÇÕES FINAIS}

A principal correlação do DM e depressão é a associação do eixo hipotálamo-pituitária-adrenal e a ativação simpática medular induzida por estresse. Os dados apontam recentes descobertas da insulina e sua sinalização associadas à neuromodulação, neuroproteção e neurotransmissão. No entanto, não existem grandes estudos com grupos de controle e randomizados que confirmem essa associação. Ademais, variáveis como atenção do médico à situações psicoemocionais devem ser investigadas, com o objetivo de melhorar o prognóstico e reduzir a morbidade dos pacientes.

\section{REFERÊNCIAS}

1. ALI S, et al. The prevalence of co-morbid depression in adults with Type 2 diabetes: a systematic review and meta-analysis. Diabet Med, 2006; 23(11): 1165-1173.

2. ANDERSON RJ, et al. The Prevalence of Comorbid Depression in Adults with Diabetes. Diabetes Care, 2001; 24(6): 1069-1078.

3. ATLANTIS E, et al. Collaborative care for comorbid depression and diabetes: a systematic review and meta-analysis. BMJ Open, 2014; 4: e004706.

4. CARNETHON MR, et al. Longitudinal Association Between Depressive Symptoms and Incident Type 2 Diabetes Mellitus in Older Adults. Arch Intern Med, 2007; 167: 802-807.

5. CIECHANOWSKI P, et al. The Relationship of Depressive Symptoms to Symptom Reporting, Self Care and Glucose Control in Diabetes. Diab Care, 2002; 25: 731-736.

6. FESTA AMD, et al. Heart Rate in Relation to Insulin Sensitivity and Insulin Secretion in Nondiabetic Subjects. Diabetes Care, 2000; 23(5): 624-628.

7. FIORE V, et al. The association between diabetes and depression: a very disabling condition. Endocrine, 2015; 48(1): 14-24.

8. GOLDEN SH, et al. Examining a Bidirectional Association Between Depressive Symptoms and Diabetes. JAMA, 2008; 299(23): 2751-2759.

9. KATON WJ, et al. A multifaceted intervention to improve treatment of depression in primary care. Arch Gen Psychiatry, $1996 ; 53: 924$.

10. KATON WJ, et al. The Association of Comorbid Depression with Mortality in Patients with Type 2 Diabetes. Diabetes Care, 2005; 28(11): 2668-2672.

11. LEEDOM L, et al. Symptoms of depression in patients with type II diabetes mellitus. Psychosomatics, 1991; 32(3): $280-286$.

12. LUSTMAN PJ, et al. Cognitive behavior therapy for depression in type 2 diabetes mellitus. A randomized, controlled trial. Ann Intern Med., 1998; 129(8): 613-21.

13. LUSTMAN PJ, et al. Depression and Poor Glycemic Control A meta-analytic review of the literature. Diabetes Care, 2000; 23: 934942.

14. LUSTMAN PJ, et al. Fluoxetine for Depression in Diabetes A randomized double-blind placebo-controlled trial. Diabetes Care, 2000; 23: 618-623.

15. MAHEUXP, et al. Fluoxetine improves insulin sensitivity in obese patients with noninsulin dependent diabetes mellitus independently of weight loss. Int J Obes Relat Metab Disord, 1997; 21:97-102.

16. MIN YU, et al. Depressão e risco de diabetes: uma meta-análise. Canadian Journal of Diabetes, 2015; 39: $266-272$.

17. MOREIRA RO, et al. Diabetes mellitus e depressão: uma revisão sistemática. Arquivos Brasileiros de Endocrinologia \& Metabologia, 2003; 47(1): 19-29.

18. MUKHERJEE N, CHATURVEDI SK. Depressive symptoms and disorders in type 2 diabetes mellitus. Current opinion in psychiatry, 2019; 32(5): 416-421.

19. MUSSELMAN DL, et al. Relationship of Depression to Diabetes Types 1 and 2: Epidemiology, Biology, and Treatment. Biol Psychiatry, 2003; 54: 317-329.

20. NOUWEN A, et al. Associações longitudinais entre depressão e complicações do diabetes: uma revisão sistemática e meta-análise. Diabetes UK, 2019; 36: 1562-1572.

21. ROY A, ROY M. Depressive symptoms in African Americans type 1 diabetics. Depress Anxiety, 2001; 13: $28-31$.

22. RUSTAD JK, et al. The relationship of depression and diabetes: pathophysiological and treatment implications. Psychoneuroendocrinology, 2011; 36(9): 1276-1286.

23. TENG CT, et al. Depression and medical comorbidity. Archives of Clinical Psychiatry, 2005; 32(3): 149-159. 